Disponível em

http://www.anpad.org.br/rac

RAC, Rio de Janeiro, v. 18, n. 4, art. 2, pp. 397-415, Jul./Ago. 2014

http://dx.doi.org/10.1590/1982-7849rac20141049

$((c))$ EY-No

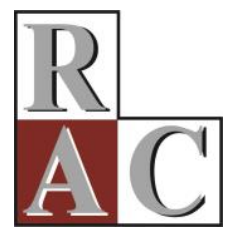

\title{
Dinâmicas do Desempenho Financeiro, da Mudança Estratégica e da Concentração Industrial em Empresas Brasileiras
}

\section{Financial Performance, Strategic Change and Industrial Concentration Dynamics in Brazilian Companies}

Renato Nogueira Starling E-mail: renatonost@yahoo.com.br Universidade de Brasília - Experimenta/UnB Campus Universitário Darcy Ribeiro, Prédio da FACE, Asa Norte, 70910-900, Brasília, DF, Brasil.

Rafael Barreiros Porto E-mail: rafaelporto@unb.br Universidade de Brasília - PPGA/UnB Campus Universitário Darcy Ribeiro, Prédio da FACE, Asa Norte, 70910-900, Brasília, DF, Brasil.

Artigo recebido em 17.08.2012. Última versão recebida em 10.02.2014. Aprovado em 11.02.2014. 


\title{
Resumo
}

O impacto da mudança estratégica da empresa em relação ao seu desempenho financeiro é um tema inconclusivo na área de gestão. Isso se deve, entre outros fatores, à não adoção de uma perspectiva dinâmica. Nesta pesquisa, investigaram-se os efeitos das mudanças estratégicas pretéritas dos conselhos de administração, dos clientes e dos produtos (considerando, para tanto, a concentração industrial representada pela quantidade de empresas no setor) sobre a lucratividade, o crescimento das vendas e o valor de mercado em empresas de capital aberto. Foram igualmente avaliados os impactos dos desempenhos passados e da concentração do setor sobre as mudanças estratégicas subsequentes. Usando dados de 68 companhias em 10 anos, construiu-se análise em painel withinenterprise, realizada através de equações de estimação generalizadas, técnica que considera a autocorrelação temporal da variável dependente. Os resultados indicaram que as mudanças estratégicas geram efeitos de curto e longo prazo nos desempenhos financeiros, e estes têm efeitos posteriores na mudança empresarial, independentes da concentração industrial. Esta, por sua vez, ora incentiva o alto desempenho e a mudança estratégica, ora os desestimula. A pesquisa contribui para explicações causais evolucionistas das relações entre o desempenho financeiro e as mudanças estratégicas.

Palavras-chave: mudança estratégica; desempenho financeiro; concentração do setor; estratégia empresarial.

\begin{abstract}
The impact a company's change in strategy has on its financial performance is an inconclusive topic in management. This is partially due to a failure to adopt a dynamic perspective. This study investigated the effects of past strategic changes in boards of directors, customers and products (taking into consideration the industrial concentration represented by the number of companies in the sector) on profitability, sales growth and market value of publicly traded companies. We also assessed the impact past performance and industry concentration has on subsequent strategic changes. Using data from 68 companies over ten years, we built up a within-enterprise panel analysis using generalized estimating equations, a technique that considers the temporal autocorrelation of the dependent variable. The results indicated that strategic changes generate short and long-term financial performances and these effects generate subsequent strategic changes, independent of industry concentration. This, in turn, sometimes encourages high financial performance and strategic change, but at other times discourages them. This research contributes to evolutionary causal explanations on the relationship between financial performance and strategic changes.
\end{abstract}

Key words: strategic change; financial performance; industry concentration; enterprise strategy. 


\section{Introdução}

A dimensão financeira do desempenho empresarial incorpora aspectos relativos à lucratividade, ao crescimento de receita e ao valor de mercado (Brito, Brito, \& Morganti, 2009; Venkatraman \& Ramanujam, 1986). O estudo dos fatores que podem influenciá-la é um dos temas mais tradicionais da área de estratégia empresarial, porém ainda é inconclusivo (Capon, Farley, \& Hoenig, 1990; Furrer, Thomas, \& Goussevskaia, 2008).

As estratégias relativas aos produtos e aos clientes, por sua vez, estão entre os critérios mais frequentemente apontados como condutores do desempenho financeiro (Capon et al., 1990). Além delas, a composição da direção e do conselho de administração da empresa vem sendo investigada como um dos seus principais determinantes (Certo, Lester, Dalton, \& Dalton, 2006; Haynes \& Hillman, 2010). Em conjunto, essas estratégias se referem, respectivamente, aos problemas empresariais e administrativos de Miles e Snow (2003) e podem explicar que tipo de estratégias tem mais impacto no desempenho financeiro.

Crescente número de estudos tem abordado a estratégia como ações e reações das organizações frente a seu ambiente competitivo, considerando a concentração do setor empresarial (Hitt, Boyd, \& Li, 2004; Hutzschenreuter \& Israel, 2009; Ketchen, Snow, \& Hoover, 2004). Nesse contexto, a empresa pode promover as mudanças estratégicas em momentos mais adequados se souber o efeito dessas mudanças (Pettigrew, 2012). Por exemplo, se souber que uma mudança administrativa tende a ter efeito negativo no valor de mercado e que o setor em que a empresa atua tem se tornado mais concentrado, ela pode promover a permanência de seu conselho de administração, o que favoreceria a estabilização do preço das ações da companhia.

Entretanto as pesquisas que consideram a dinâmica das mudanças de produtos, clientes e alta administração como causas ou consequências dos desempenhos raramente consideram mais do que um desses aspectos. Nesta pesquisa ${ }^{(1)}$, as mudanças estratégicas foram tratadas de maneira integrada como condutoras da lucratividade, do crescimento de vendas e do valor de mercado das empresas. Como os resultados de ações passadas podem influenciar ações futuras, elas também foram abordadas como consequências de desempenhos obtidos em períodos anteriores. Para analisar essas relações, este estudo utilizou dados de diversas empresas, dentro de alguns setores, em uma série de anos, incluindo o uso de dados defasados para verificar relações temporais entre as variáveis estudadas.

O objetivo da pesquisa foi, portanto, analisar a dinâmica entre o desempenho financeiro e as mudanças estratégicas, controlando a concentração setorial, em empresas brasileiras de capital aberto. Para tanto, foram testadas, inicialmente: (a) a influência das mudanças estratégicas pretéritas de conselho de administração, produtos e clientes, considerando as mudanças da concentração industrial, sobre as dimensões do desempenho corrente, a saber: a lucratividade, o crescimento em vendas e o valor de mercado; e, posteriormente, (b) a influência das alterações da concentração da indústria e dessas dimensões do desempenho pretéritas sobre as mudanças estratégicas de conselho de administração, produtos e clientes em tempos correntes. Foram elaborados modelos e estes foram testados por meio de equações de estimação generalizadas, técnica de regressão semiparamétrica, que calcula a resposta média da população quando há autocorrelação temporal na variável dependente e faz estimação dentro de cada empresa por setor (painel de empresas).

Na sequência deste artigo são abordadas as dimensões que se adotaram como representativas do desempenho financeiro e sua relação como consequências ou causas das mudanças estratégicas empresariais. Em seguida, constam os modelos usados na pesquisa e o método adotado. Feito isso, os resultados são apresentados e discutidos e, por fim, são destacadas as principais contribuições da pesquisa, bem como as limitações que esta apresenta. 


\section{Desempenho Financeiro Como Consequência e Causa das Mudanças Estratégicas}

O desempenho empresarial remete à consecução de um objetivo da organização (Lebas, 1995) e é tratado como uma variável dependente de fatores estratégicos empresariais (Capon et al., 1990). Ele vem sendo operacionalizado por diversas métricas (Lebas, 1995) via dois grandes domínios de indicadores: financeiros e operacionais (Venkatraman \& Ramanujam, 1986). Restringindo o escopo ao desempenho financeiro, Combs, Crook e Shook (2005) identificaram três dimensões desse construto na literatura: lucratividade, crescimento em vendas (ou receita) e valor de mercado.

A lucratividade é medida por meio da margem de lucro, do lucro por ação, do retorno sobre o ativo e/ou do retorno sobre o capital próprio (Gitman, 2004). Contudo ela é incapaz de representar aspectos incorporados ao rendimento econômico, como a distribuição da rentabilidade no tempo, os fluxos de caixa e o risco. Esses aspectos são incorporados pela avaliação feita pelo mercado financeiro do valor da empresa (Gitman, 2004). Nesse sentido, existem argumentações que defendem a ideia centenária de considerar o aumento da riqueza do acionista como o principal objetivo do administrador (Araújo \& Assaf, 2003; Sundaram \& Inkpen, 2004).

Por sua vez, o crescimento empresarial pode ser aferido por meio de diversas métricas (Gupta, Guha, \& Krishnaswami, 2013), tais como o crescimento da produção, o crescimento das vendas ou o aumento do número de empregados da empresa. Entre elas, o crescimento de vendas é diretamente mensurado via receita, sendo a apropriação pela empresa daquilo que ofertou aos consumidores, sem considerar o custo total (Brito et al., 2009).

$\mathrm{O}$ argumento de que a organização precisa mudar sua estratégia para se adaptar às condições ambientais do momento é frequentemente encontrado na literatura (Chandler, 1962; Miles \& Snow, 2003). A mudança tem surgido como um processo que conduz ao desempenho financeiro empresarial. Pettigrew (2012) sugere que o processo de mudança seria composto pelas ações, reações e interações entre diversas partes interessadas que levam a organização de um estado presente a um estado futuro, capazes de produzir efeitos de segunda ordem ou múltiplas consequências.

Miles e Snow (2003), em 1978, atentaram para os efeitos que variáveis ambientais produziam sobre as empresas e delinearam a maneira como essas empresas reagiriam. Os autores identificaram a existência de um ciclo adaptativo composto por três problemas: (a) o problema empresarial diz respeito ao mercado em que a empresa irá atuar, e aos produtos oferecidos para esse mercado; (b) o problema de engenharia aborda os meios pelos quais a empresa produz os bens escolhidos; e (c) o problema administrativo é relativo à estabilização das soluções encontradas por meio de uma institucionalização da gestão da empresa. Segundo eles, alterações ambientais induzem mudanças em pelo menos um desses três problemas, o que exige que a empresa se adapte. Neste estudo, optou-se por abordar alguns aspectos da mudança relativos ao problema empresarial e ao problema administrativo.

O problema empresarial é tratado por meio das mudanças estratégicas de produtos e clientes. E, conforme Miles e Snow (2003), em empresas marcadas por longa continuidade no tempo, o ciclo adaptativo da empresa costuma ser ativado por meio do problema empresarial, pois os demais já se encontram razoavelmente resolvidos. Assim, as mudanças ambientais exigem, inicialmente, adaptações nos produtos e mercados da empresa e são essas adaptações que desencadeiam mudanças tecnológicas e administrativas.

A mudança estratégica de produtos pode representar tanto uma inovação (Organização para a Cooperação Econômica e Desenvolvimento [OCDE], 2012) quanto uma alteração da participação deles no portfólio da empresa. Encontram-se relatos de que inovações de produto são determinantes de diversas dimensões do desempenho (Brito et al., 2009; Calantone, Harmancioglu, \& Droge, 2010; Capon et al., 1990). Porém exigem investimentos de retorno incerto (Sorenson, 2000), o que aumenta o risco da organização. A alteração da participação dos produtos da empresa na sua receita também é vista como uma mudança adaptativa, que pode ser benéfica para o desempenho da empresa e não implica em tantos riscos (Miles \& Snow, 2003; Nelson \& Winter, 2005). Ela representa o quanto os produtos podem 
ser adaptados para atender aos compradores em potencial ou às obrigações legais (Vrontis, Thrassou, \& Lamprianou, 2009).

Uma mudança de clientes, por sua vez, pode representar a entrada de um novo membro na carteira da empresa ou um reposicionamento de clientes com os quais ela já mantenha relações. A literatura sobre o valor do cliente no tempo sugere que a lealdade dele é um meio de melhorar o desempenho financeiro da empresa, especialmente quando se alonga o relacionamento daqueles que possibilitam melhores retornos (Ramaswami, Srivastava, \& Bhargava, 2009; Ryals \& Knox, 2005). Do ponto de vista empírico, quando a relação entre lealdade e desempenho financeiro foi testada, encontrou-se uma relação positiva entre os indicadores (Gee, Coates, \& Nicholson, 2008; Hallowell, 1996). A longo prazo, a mudança da carteira de clientes pode indicar perdas financeiras ou redirecionamento para clientes mais rentáveis (Petersen et al., 2009).

Já a mudança estratégica da composição do conselho de administração possibilita a adaptação ao ambiente (Pugliese et al., 2009), o controle do resultado empresarial e as decisões do rumo da empresa (Minichilli, Zattoni, \& Zona, 2009). A sua composição é capaz de influenciar a escolha do presidente da empresa ou as ações do corpo diretivo (Haynes \& Hillman, 2010; Quigley \& Hambrick, 2012; Westphal \& Fredrickson, 2001). Ainda, o nível de governança da empresa influencia na confiança dos investidores e, consequentemente, no valor de mercado da empresa (Andrade, Salazar, Calegário, \& Silva, 2009).

Dessa maneira, algumas teorias foram propostas para explicar como ocorre o processo de mudança estratégica nas organizações. Nelson e Winter (2005) e Quinn (1978) buscaram explicá-la como um processo que tende a ser mais incremental que revolucionário. Cyert e March (1963) apontaram a retroalimentação dada pela consecução ou não dos objetivos definidos na organização como um elemento central para determinar as mudanças incrementais.

A partir de então, os estudos começaram a tratar o desempenho não apenas como uma consequência das ações da organização, mas também como um fator explicativo delas (Greve, 2007; Ketchen \& Palmer, 1999; Labianca, Fairbank, Andrevski, \& Parzen, 2009). A lógica dessa abordagem é que a organização, apresentando um desempenho ruim, tenderia a implementar mudanças no sentido de reverter a situação em um momento seguinte (Ketchen \& Palmer, 1999) ou, apresentando um desempenho bom, reforçaria a continuidade da estratégia porque ela propicia adaptação ao ambiente (Vella \& Foxall, 2011).

Além de aspectos da história da empresa, as características do ambiente são fatores que influenciam o desempenho financeiro (Chandler, 1962; Cyert \& March, 1963; Miles \& Snow, 2003; Quinn, 1978). A teoria econômica destaca a existência de diferentes estruturas de mercado concorrência perfeita, monopólio, oligopólio e concorrência monopolística (Simonsen, 1979) - que podem estimular o bom ou o mau desempenho. Altas taxas de concentração estão relacionadas ao maior valor de mercado das empresas quando a concentração permite restringir entrada de novos competidores no setor ou no grupo estratégico, dessa forma, gerando ineficiências no mercado como o conluio (Bruce \& Marginson, 2013). Porém a desconcentração gera eficiências na competitividade empresarial por meio dos produtos levados ao mercado e clientes atendidos, que refletem em maiores vendas e lucros (Bharadwaj \& Varadarajan, 2005). Nesse sentido, a concentração setorial não é uma variável causal (Levitt \& List, 2009), mas sim contextual, que oferece condições necessárias e, em alguns casos, suficientes para que algumas ações (eficientes/ineficientes) ocorram e gerem resultados financeiros. Na próxima seção é formalizado um modelo que consolida essas perspectivas teóricas.

\section{Modelo da Pesquisa}

Os efeitos da mudança estratégica da empresa ao longo dos anos podem ser positivos ou negativos, conforme prevaleça um ou outro aspecto da mudança, independente das estruturas de 
mercado. Alguns autores têm esclarecido essas possíveis relações, sendo todas contextualizadas para o modelo da presente pesquisa.

Dessa maneira, espera-se que uma mudança estratégica dos produtos possa ser positiva na lucratividade se decorrente de uma adaptação bem-sucedida no mercado (Calantone, Cavusgil, Schmidt, \& Shin, 2004). Contudo essa mudança pode ter efeito negativo de longo prazo quando o mercado é dinâmico e uma modificação do produto no passado não atenda mais o mercado em tempo corrente (Calantone, Kim, Schmidt, \& Cavusgil, 2006). Quanto à mudança de clientes, há predições que venham a ter efeitos negativos sobre o retorno e o valor da empresa (Ramaswami et al., 2009), visto que a permanência de clientes (lealdade) reduz custos e gera expectativa futura de valor. Logo, espera-se que mudanças de clientes tenham impactos negativos nesses indicadores de desempenho financeiro.

Há indícios de que a mudança no conselho de administração é favorável ao crescimento da empresa em termos de receita de curto e longo prazos, porque novos conselheiros estão mais abertos às mudanças necessárias para se adaptar ao ambiente (Pugliese et al., 2009) e podem beneficiar empregados e executivos (David, O'Brien, Yoshikawa, \& Delios, 2010). Porém essas mudanças podem gerar efeito negativo no valor da empresa no longo prazo, por sinalizar falta de engajamento dos conselheiros anteriores (Minichilli et al., 2009). Além disso, a mudança do conselho pode produzir resultados na lucratividade no curto e no longo prazos. Fahlenbrach, Low e Stulz, (2010) demonstram que um novo conselho ou novos membros, em especial novos CEOs no conselho, recomendam resultados rápidos na lucratividade, mas, pela inexperiência (Minichilli et al., 2009), aquilo recomendado pode gerar resultados financeiros de longo prazo danosos. Então, espera-se que, no curto prazo, mudanças no conselho gerem resultados positivos e, no longo prazo, negativos.

Ademais, em setores desconcentrados, independente das mudanças estratégicas, pois há aumento na lucratividade a longo prazo, devido ao ambiente benéfico para que haja eficiências derivadas da maior competitividade empresarial (Bruce \& Marginson, 2013). Entretanto, nesses setores, podem ocorrer decréscimos no crescimento empresarial no longo prazo porque a concorrência cria limites para que as empresas cresçam, já que precisam ratear o volume que vendem entre os concorrentes (Hill, Kelly, \& Highfield, 2010). Os setores mais desconcentrados também podem ser maléficos sobre o valor de mercado da empresa a curto prazo, porque as ações empresariais caem quando há possibilidade de comparação de desempenho entre empresas similares. Mas é possível que as empresas que se sobressaem dentro de setores desconcentrados gerem maior valor ao acionista no longo prazo, por provar suas competências competitivas e não apenas por questões de boa governança (Giroud \& Mueller, 2011). Portanto, a desconcentração do setor deve impactar esses indicadores de desempenho nos sentidos previamente relatados.

Diante disso, propõem-se testar o modelo apresentado graficamente na Figura 1. Considerando que os efeitos de uma variável explicativa devem anteceder a resposta na variável dependente, optou-se por avaliar a relação no ano em que as duas observações são tomadas e com defasagens de um (t-1) e dois anos (t-2). 


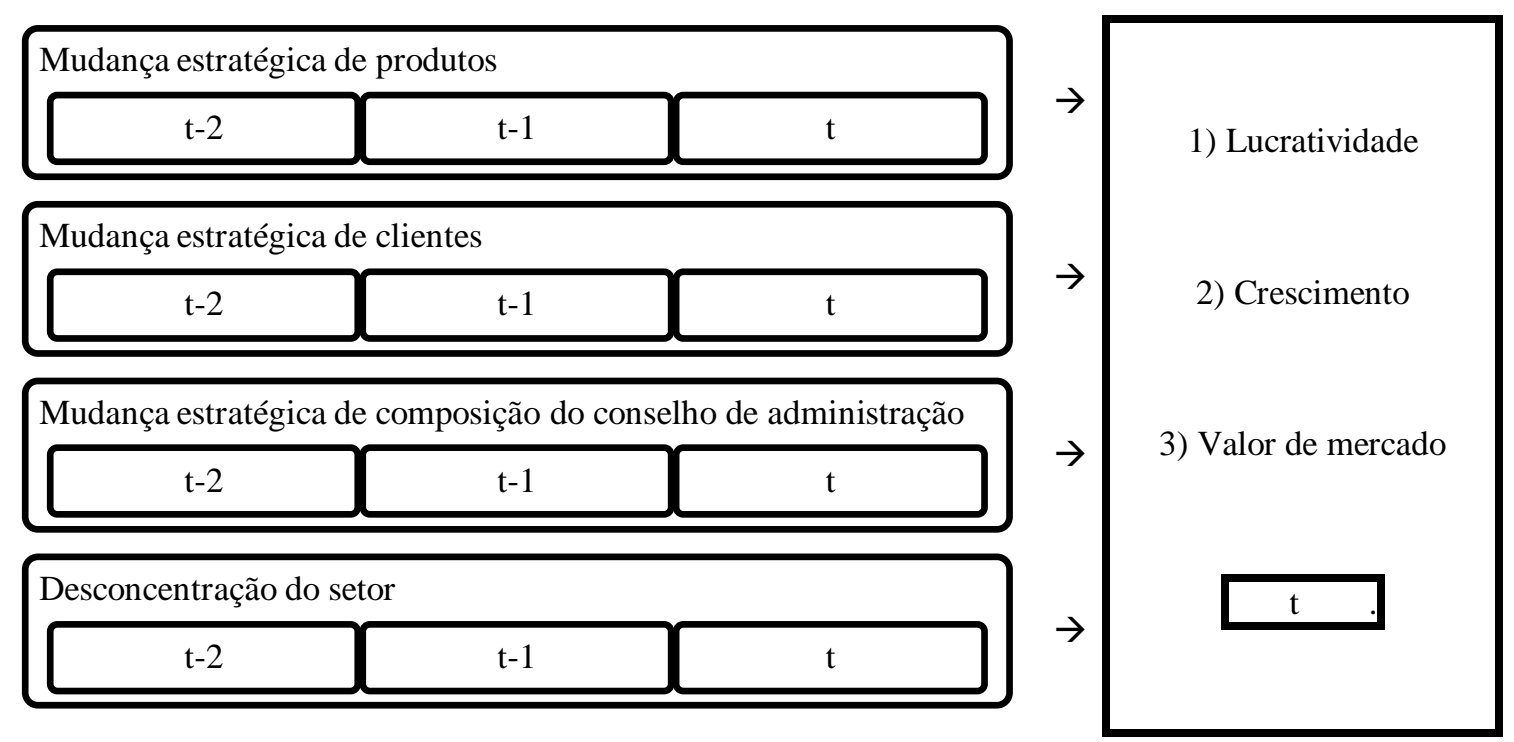

Figura 1. Modelo Explicativo do Desempenho Financeiro.

Já a relação contrária, o desempenho financeiro como gerador (ou inibidor) de mudanças, é subpesquisada na academia sem apontar uma direção especificamente clara. Mas, com base na proposta teórica de Cyert e March (1963), espera-se que os índices de mudanças estratégicas sejam explicados por desempenhos anteriores. Bons desempenhos lucrativos anteriores estão associados a baixo monitoramento pelo conselho de administração (Pugliese, Minichilli, \& Zattoni, 2013). Isso pode levar às mudanças do próprio conselho, por não cumprir um de seus papéis básicos. Já o alto crescimento em vendas prévio pode fazer com que a empresa busque alterar clientes, diminuindo sua dependência em relação a alguns clientes não rentáveis (Bijmolt et al., 2010). Ao mesmo tempo, pode levar à estabilização do conselho de administração do tipo relacional, que tende a adotar ações em prol dos executivos e funcionários e, assim, perpetuar-se (David et al., 2010).

Altos valores de mercado antecedentes podem capitalizar a troca de recursos necessários para inovações e adaptações dos produtos no mercado (Sood \& Tellis, 2009) e, dessa forma, possibilitar maior lucratividade no futuro. Além disso, podem incentivar a alteração de conselhos de administração se estes obtiverem baixa aprovação de votos. Essa baixa aprovação está associada à baixa recompensa para CEOs, melhores aquisições de empresas e melhores desinvestimentos (Fischer, Gramlich, Miller, \& White, 2009). Além do mais, as mudanças estratégicas não ocorrem no vácuo, portanto, dimensões setoriais podem afetá-las. A estrutura de mercado é um contexto que pode facilitar as mudanças, em especial, de clientes a longo prazo. Quanto maior o número de empresas atuantes no mercado, maior a oferta competitiva e isso, por sua vez, gera o contexto ideal para a deslealdade de clientes. Empresas podem vivenciar esse fenômeno em períodos posteriores e sua base de clientes favoritos (os que geram maior receita) pode mudar (Wright \& Riebe, 2010). Em compensação, setores mais concentrados tendem a ter maior heterogeneidade de expertise do CEO, que, por sua vez, está relacionada com baixo valor de mercado e baixa importância de seus apontamentos, podendo induzir uma mudança no conselho para atenuar esse problema (Knyazeva, Knyazeva, \& Raheja, 2009). A Figura 2 apresenta todas essas possibilidades. Optou-se por avaliar as relações com defasagens de um (t-1) e dois anos (t-2), com exceção da desconcentração setorial que pode ter influência defasada e também corrente. 


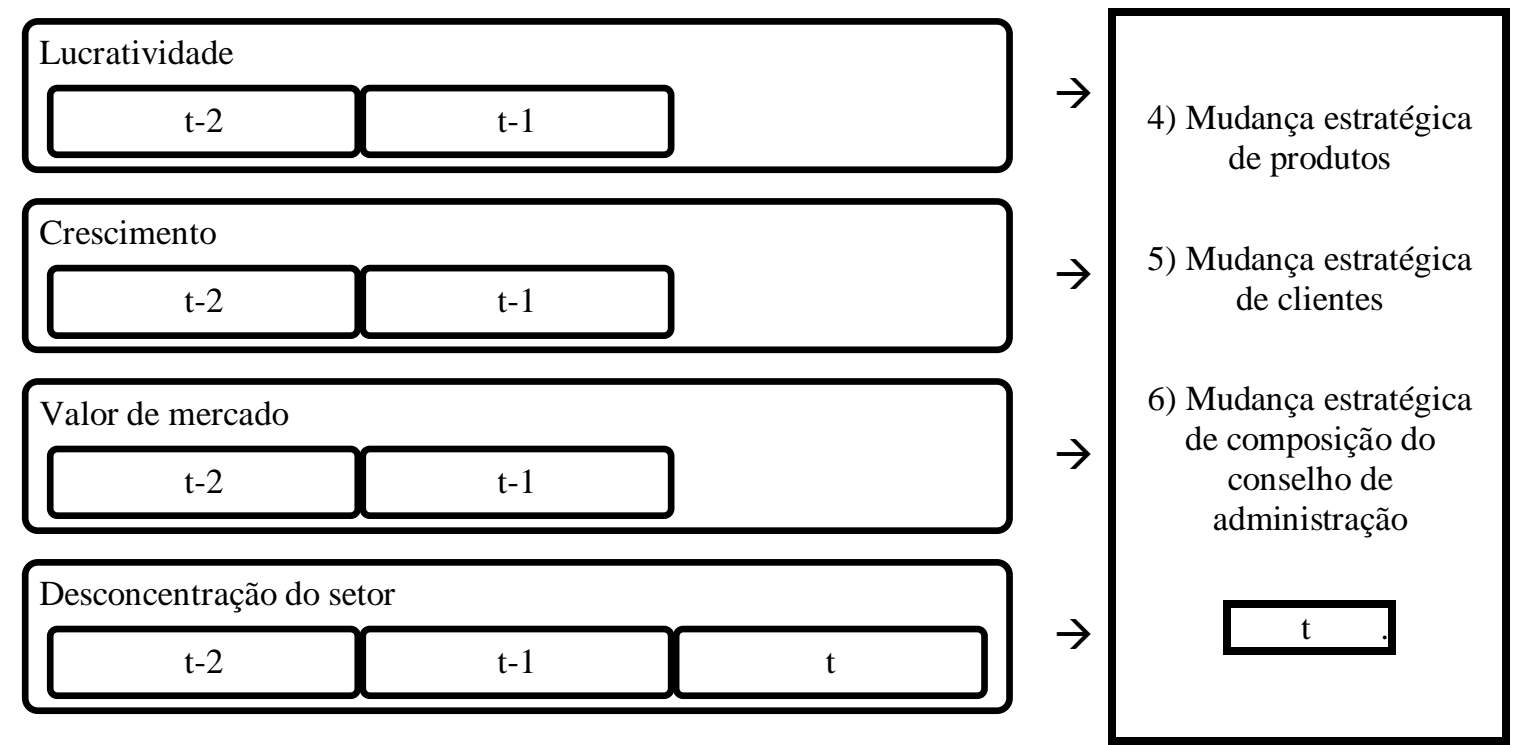

Figura 2. Modelo Explicativo da Mudança Estratégica.

\section{Método}

\section{Fontes de dados e amostra}

A principal fonte de informação foi a base de dados da Comissão de Valores Mobiliários (CVM) referente ao período de 1999 e 2008. Adicionalmente, utilizaram-se dados disponibilizados pelo Instituto Brasileiro de Geografia e Estatística (IBGE) e pela consultoria Economática. Os dados da CVM referentes aos produtos e clientes não são disponibilizados de maneira padronizada, o que limitou o tamanho da amostra para 68 empresas ao longo de 10 anos, totalizando 662 observações (combinação ano e empresa por setor). Para cada empresa, foram contadas, em média, 9,74 observações anuais das variáveis dependentes $($ D.P. $=1,11)$. O teste do poder amostral de 12 preditores foi igual a $83 \%$, o suficiente para reduzir o Erro Tipo 2.

As empresas pertenciam aos seguintes setores (Divisão da Classificação Nacional de Atividades Econômicas [CNAE]): agricultura, pecuária e serviços relacionados; alojamento; armazenamento e atividades auxiliares dos transportes; captação, tratamento e distribuição de água; comércio e reparação de veículos automotores e motocicletas; confecção de artigos do vestuário e acessórios; edição e edição integrada à impressão; eletricidade, gás e outras utilidades; fabricação de celulose, papel e produtos de papel; fabricação de coque, de produtos derivados do petróleo e de biocombustíveis; fabricação de equipamentos de informática, produtos eletrônicos e ópticos; fabricação de máquinas e equipamentos; fabricação de máquinas, aparelhos e materiais elétricos; fabricação de produtos alimentícios; fabricação de produtos de metal, exceto máquinas e equipamentos; fabricação de produtos de minerais não metálicos; fabricação de produtos diversos; fabricação de produtos químicos; fabricação de produtos têxteis; fabricação de veículos automotores, reboques e carrocerias; metalurgia; obras de infraestrutura e, finalmente, transporte terrestre.

\section{Procedimentos de coleta e análise dos dados}

Os dados foram extraídos por meio do Sistema de Divulgação Externa ITR/DFP/IAN da CVM. No formato original, a empresa declara livremente o nome do produto e do cliente. Por isso, foi necessário padronizar diferentes grafias do mesmo objeto ou eliminar erros ortográficos. Com essas exceções, qualquer informação de conteúdo diferente foi tratada como produtos ou clientes diferentes. 
As informações sobre produtos oferecidos pela empresa apresentavam seus nomes e o percentual que cada um deles representava para a receita da empresa no ano. Por exemplo, em uma determinada siderúrgica, os aços para construção mecânica representavam $83 \%$ da receita em 2008, e os cilindros para laminação, 17\%. No ano anterior, essas proporções eram, respectivamente, $79 \%$ e $21 \%$. As informações relativas aos clientes apresentam formato semelhante, e as relativas à composição do conselho de administração informam o nome e o número do Cadastro de Pessoas Físicas (CPF) de cada conselheiro.

Adicionalmente, o Cadastro Nacional da Pessoa Jurídica (CNPJ) de cada empresa foi consultado no site da Receita Federal do Brasil para a identificação da Classificação Nacional de Atividades Econômicas (CNAE) da atividade principal de cada empresa. A partir dessa informação, recorreu-se ao site do IBGE para obter os dados referentes à concentração do setor. Por fim, recolheram-se os dados referentes ao valor de mercado das empresas da amostra na base de dados Economática.

Foi adotada uma variável para representar cada dimensão do desempenho. A lucratividade foi indicada pelo retorno sobre o ativo (ROA), que é o retorno percentual obtido pelo capital investido em ativos na empresa. O crescimento das vendas (VEN) foi calculado pela razão entre as vendas da empresa no ano corrente em relação às vendas no ano anterior. O valor de mercado (VAM) foi extraído diretamente do banco de dados da Economática. Nas duas últimas variáveis, trabalhou-se com valores deflacionados, como mostram as Fórmulas 1, 2 e 3.

$$
\begin{aligned}
& \mathrm{ROA}_{\mathrm{e}, \mathrm{t}}=\frac{\text { Lucro Líquido do Exercício }_{\mathrm{e}, \mathrm{t}}}{\text { Ativo Total }} \\
& \mathrm{VEN}_{\mathrm{e}, \mathrm{t}}=\left(\frac{\text { Receita bruta de vendas }_{\mathrm{e}, \mathrm{t}}}{\text { Receita bruta de vendas }_{\mathrm{e}, \mathrm{t}-1}} \mathrm{X}_{\text {deflator }} \text { d }_{\mathrm{t}}\right)-1 \\
& \text { VAM }_{\mathrm{e}, \mathrm{t}}=\text { Valor de mercado }_{\mathrm{e}, \mathrm{t}} \mathrm{x} \text { deflator }_{\mathrm{t}}
\end{aligned}
$$

Em que o índice e representa cada empresa e $\mathbf{t}$ indica cada ano da série.

Para indicar a mudança estratégica, foram criados três índices que refletem a mudança na participação de cada produto (PRO) ou cliente (CLI) na receita da empresa, ou a mudança ocorrida em seu conselho de administração (CAD) entre dois momentos. As Fórmulas 4, 5 e 6 apresentam as fórmulas dessas métricas.

$$
\begin{aligned}
& \mathrm{PRO}_{\mathrm{e}, \mathrm{t}}=\sqrt{\sum_{\mathrm{i}=1}^{\mathrm{n}}\left(\mathrm{P}_{\mathrm{i}_{\mathrm{e}, \mathrm{t}}}-\mathrm{P}_{\mathrm{i}_{\mathrm{e}, \mathrm{t}-1}}\right)^{2}} \\
& \mathrm{CLI}_{\mathrm{e}, \mathrm{t}}=\sqrt{\sum_{\mathrm{i}=1}^{\mathrm{n}}\left(\mathrm{C}_{\left.\mathrm{i}_{\mathrm{e}, \mathrm{t}}-\mathrm{C}_{\mathrm{i}_{\mathrm{e}, \mathrm{t}-1}}\right)^{2}}\right.} \\
& \mathrm{CAD}_{\mathrm{e}, \mathrm{t}}=1-\frac{\left|\mathrm{A}_{\mathrm{e}, \mathrm{t}} \cap \mathrm{A}_{\mathrm{e}, \mathrm{t}-1}\right|}{\left|\mathrm{A}_{\mathrm{e}, \mathrm{t}}\right|}
\end{aligned}
$$

\section{Em que:}

i: cada produto ou cliente destacado pela empresa como componente de sua receita no formulário de informações anuais da CVM; n: número de produtos ou clientes destacados pela empresa como componentes de sua receita no formulário de informações anuais da CVM; $\mathrm{P}_{\mathrm{ie}, \mathrm{t}}$ e $\mathrm{C}_{\mathrm{ie}, \mathrm{t}}$ : percentual de participação do produto ou cliente $\mathbf{i}$ (respectivamente) na composição da receita da empresa e no ano t; $\mathrm{P}_{\mathrm{ie}, \mathrm{t}-1}$ e $\mathrm{C}_{\mathrm{ie}, \mathrm{t}-1}$ : percentual de participação do produto ou cliente $\mathbf{i}$ (respectivamente) na composição da receita da empresa e no ano t-1; $A_{e, t}$ : Conjunto dos membros do conselho de administração da empresa 
e no ano t; $A_{e, t-1}$ : Conjunto dos membros do conselho de administração da empresa e no ano t-1; $\cap$ : Interseção de conjuntos; $|\mathrm{A}|$ : Cardinalidade (número de elementos) de um conjunto $\mathbf{A}$.

Para CLI e PRO, considera-se relevante o produto ou o cliente que conste individualmente identificado no formulário de informações anuais da empresa, sendo possível apurar sua participação.

A alteração da estrutura do setor é representada pela proporção de grandes empresas que atuam no setor, no tempo. São consideradas grandes as empresas com mais de 500 funcionários. É apurado pelo indicador:

$$
\operatorname{DESCON}_{s, t}=\frac{\text { número de grandes empresas }}{s, t}
$$

Em que DESCON ${ }_{\mathrm{s}, \mathrm{t}}$ indica a desconcentração do setor $\mathbf{s}$ no ano $\mathbf{t}$ e o setor é definido pela divisão da CNAE.

Em todos os casos, as variáveis sofrem duas transformações para serem inseridas na análise. Primeiramente, dado que as empresas atuam em setores distintos, que podem apresentar comportamentos particulares para cada variável, optou-se por trabalhar com valores relativos do indicador frente à média do setor. Assim, cada valor foi dividido pelo valor médio de todas as empresas daquele setor, em todos os anos da amostra. Isso padroniza os parâmetros de todas as análises, permitindo comparação proporcional. Portanto, se o valor de cada variável for igual a um, significa que esse valor, naquele ano, está igual a média das empresas do setor. Se estiver abaixo (acima) de um, está menor (maior) que a média anual das empresas do setor. Em seguida, dada a distribuição observada das variáveis, foi calculado o logaritmo de cada indicador, com o fim de deixá-las com distribuição normal. Dessa forma, os valores que eram iguais a um passam a ser zero (média do setor). Números positivos passam a ser acima da média e números negativos, abaixo da média.

A Tabela 1 elenca as estatísticas descritivas das variáveis já transformadas para os dados em painel anual de empresas dentro do setor, fazendo com que os desvios padrões sejam baixos em relação à média. Existiram muitas empresas que apresentaram mudanças anuais de produto e de clientes, taxas de crescimento anual da empresa e valores anuais de mercado da empresa abaixo da média do setor, tornando a média negativa. Havia também muitas empresas que apresentaram mudanças anuais do conselho de administração e de sua lucratividade acima da média do setor, tornando a média positiva. Como um todo, vários setores eram mais concentrados do que desconcentrados (média negativa), mas anualmente variava pouco a estrutura do mercado desses setores (baixo desvio padrão).

Tabela 1

Análise Descritiva das Variáveis Utilizadas na Pesquisa

\begin{tabular}{ccc}
\hline Variável & Média & Desvio Padrão \\
\hline PRO & $-0,25$ & 0,06 \\
CLI & $-0,35$ & 0,04 \\
CAD & 0,43 & 0,04 \\
DESCON & $-0,90$ & 0,02 \\
ROA & 0,62 & 0,05 \\
VEN & $-1,65$ & 0,07 \\
VAM & $-2,62$ & 0,05 \\
\hline
\end{tabular}

As métricas adotadas, com as transformações já apresentadas (situação em relação à média do setor e conversão em logaritmo), atenderam aos pressupostos da normalidade (todos testes Kolmogorov- 
Smirnov não foram significativos $\mathrm{p}>0,05$ ), multicolinearidade (VIF variou entre 1,8 a 2,1) e homocedasticidade (gráfico dos resíduos e valores ajustados não apresentaram padrão específico). Contudo, há correlação serial nas variáveis dependentes, o que exigiu fazer análises das matrizes de correlação de trabalho (Ballinger, 2004). Estas não apresentaram um padrão específico para cada análise. Dessa forma, optou-se pela matriz de correlação não estruturada, a melhor quando não há um padrão específico. Todos os resultados relatados são baseados nessa matriz de correlação de trabalho. Houve checagem do critério de quase verossimilhança sob o modelo de independência (QIC) entre os modelos com as variáveis independentes versus modelos nulos (apenas a constante). Em todos os modelos, os indicadores QIC foram mais ajustados para os modelos com as variáveis independentes. O QIC apresentou-se melhor para a não estruturada em todas as análises. Logo, todos os resultados relatados são baseados nessa matriz de correlação de trabalho.

Entre os modelos estatísticos para dados em painel, optou-se por adotar as equações de estimação generalizadas, técnica de regressão semiparamétrica que calcula a resposta média da população quando há autocorrelação temporal na variável dependente e faz estimação dentro de cada grupo (empresa por setor). Essa técnica permite diferentes distribuições na variável dependente e uma função diferenciável como ligação entre as variáveis dependentes e a independente, como nos Modelos Lineares Generalizados (Ballinger, 2004). Além disso, estende a análise dessa técnica para conjuntos de dados repetidos (diversos valores de cada variável para a mesma empresa, um em cada ano). Uma vez que os dados foram transformados em logaritmos, adotou-se um modelo de resposta linear. Tendo em vista o modelo teórico, foram realizados testes com efeitos principais.

Cada um dos modelos propostos foi comparado com um modelo explicativo mais simples, em que apenas as variáveis de controle (empresa e ano) foram usadas para explicar o fenômeno. Comparando cada modelo com seu correspondente simplificado (modelo base), constataram-se melhores ajustes, medidos pelo QICC (critério de quase verossimilhança sob o modelo de independência corrigido). Por isso, nos resultados, apenas os modelos de trabalho são relatados.

O cuidado de considerar a variável explicativa não apenas no mesmo ano do seu efeito, mas também com defasagem em até dois anos antecedentes, associado aos controles adotados e às relações de covariação entre as variáveis detectadas pela técnica estatística, permite que se façam inferências de causalidade nas relações entre as variáveis independentes e dependentes, caracterizando o método como quase experimental.

\section{Resultados}

Nesta seção, são apresentados os resultados de impacto sobre o desempenho financeiro (Figura 1) e, em seguida, passa-se à apresentação dos impactos sobre a mudança estratégica (Figura 2).

Os efeitos anuais das variáveis independentes sobre o desempenho financeiro para cada empresa em seus respectivos setores (Figura 1) foram melhor ajustados para o valor de mercado $\left(\mathrm{R}^{2}\right.$ médio $=$ $35,5 \%)$, lucratividade $\left(\mathrm{R}^{2}\right.$ médio $\left.=32,5 \%\right)$ e crescimento de venda $\left(\mathrm{R}^{2}\right.$ médio $\left.=19,9 \%\right)$, respectivamente. Os dados foram bem ajustados para algumas empresas, mas, para a maior parte delas, não. A Tabela 2 apresenta os resultados, incluindo exemplos de empresas que obtiveram maior impacto no desempenho. Percebe-se que os efeitos correntes e defasados da mudança de produtos foram impactantes apenas sobre a lucratividade, sendo t e t-1 com efeitos positivos e t-2, negativos. Dessa forma, no agregado, quanto maior a mudança de produtos como composição das receitas, maior a lucratividade, com os efeitos defasados maiores do que os correntes. Igualmente, observa-se que apenas o efeito defasado em t- 1 da mudança de cliente teve impacto negativo sobre a lucratividade e seu efeito corrente em $\mathrm{t}$ teve impacto também negativo sobre o valor de mercado. Assim, quanto maior a mudança de clientes como composição das receitas, menor o valor de mercado no ano corrente e menor a lucratividade um ano após. 
É igualmente possível notar que as mudanças no conselho de administração têm impacto de curto e longo prazos em todos os indicadores financeiros. No agregado, os efeitos sobre a lucratividade são negativos. Os efeitos sobre o crescimento em vendas são positivos, em especial o de curto prazo, e os efeitos sobre o valor de mercado são sempre negativos.

Quanto mais desconcentrado o setor, mais benéfico para o aumento da lucratividade, especialmente ao considerar o longo prazo, e menos benéfico para o crescimento de vendas, considerando o efeito nefasto em dois anos após a mudança no setor. Sobre o valor de mercado, a desconcentração do setor parece ser o contexto menos propício para seu aumento, sobretudo no momento $t$ (corrente), mas como há efeitos benéficos da desconcentração do setor a longo prazo (t-1), este atenua o efeito negativo daquele.

Tabela 2

Impacto das Mudanças Estratégicas no Desempenho Empresarial

\begin{tabular}{|c|c|c|c|c|c|c|}
\hline \multirow{2}{*}{$\begin{array}{l}\text { Variável } \\
\text { preditora }\end{array}$} & \multicolumn{2}{|c|}{ Retorno sobre o ativo ${ }^{a}$} & \multicolumn{2}{|c|}{ Crescimento das vendas $^{b}$} & \multicolumn{2}{|c|}{ Valor de mercado ${ }^{c}$} \\
\hline & Estimativa & Erro padrão & Estimativa & Erro padrão & Estimativa & Erro padrão \\
\hline $\mathrm{PRO}(\mathrm{t})$ & $0,13^{*}$ & 0,06 & $-0,32$ & 0,17 & $-0,05$ & 0,03 \\
\hline $\mathrm{PRO}(\mathrm{t}-1)$ & $0,36^{* *}$ & 0,04 & $-0,11$ & 0,12 & 0,03 & 0,07 \\
\hline $\mathrm{PRO}(\mathrm{t}-2)$ & $-0,12 *$ & 0,06 & 0,22 & 0,17 & 0,04 & 0,05 \\
\hline $\operatorname{CLI}(\mathrm{t})$ & $-0,03$ & 0,06 & 0,16 & 0,17 & $-0,12 *$ & 0,05 \\
\hline $\operatorname{CLI}(\mathrm{t}-1)$ & $-0,13^{*}$ & 0,06 & 0,25 & 0,31 & $-0,07$ & 0,13 \\
\hline $\operatorname{CLI}(\mathrm{t}-2)$ & 0,02 & 0,05 & 0,32 & 0,38 & $-0,04$ & 0,05 \\
\hline $\mathrm{CAD}(\mathrm{t})$ & $0,19^{*}$ & 0,09 & $0,85^{* *}$ & 0,23 & $-0,18^{*}$ & 0,08 \\
\hline $\mathrm{CAD}(\mathrm{t}-1)$ & 0,09 & 0,05 & $0,47 *$ & 0,21 & $-0,14$ & 0,11 \\
\hline $\mathrm{CAD}(\mathrm{t}-2)$ & $-0,24 * *$ & 0,04 & 0,02 & 0,16 & $-0,23^{* *}$ & 0,07 \\
\hline $\operatorname{DESCON}(\mathrm{t})$ & 0,04 & 0,23 & 0,16 & 0,71 & $-0,61 * *$ & 0,11 \\
\hline $\operatorname{DESCON}(\mathrm{t}-1)$ & $0,76^{* *}$ & 0,18 & 0,65 & 0,46 & $0,36^{*}$ & 0,17 \\
\hline DESCON(t-2) & $0,41 * *$ & 0,14 & $-1,82 *$ & 0,91 & 0,23 & 0,26 \\
\hline $\mathrm{R}^{2}$ Linear médio & \multicolumn{2}{|c|}{$32,50 \%$} & \multicolumn{2}{|c|}{$19,90 \%$} & \multicolumn{2}{|c|}{$35,50 \%$} \\
\hline \multirow{3}{*}{$\begin{array}{l}\text { Três empresas } \\
\text { com maior } \\
\text { impacto }\left(\mathrm{R}^{2}\right. \\
\text { linear) em seus } \\
\text { respectivos } \\
\text { setores }\end{array}$} & \multicolumn{2}{|c|}{$\begin{array}{l}\text { Cecrisa Revestimento } \\
\text { Cerâmicos S.A. }(73,2 \%)\end{array}$} & \multicolumn{2}{|c|}{$\begin{array}{c}\text { Gradiente eletrônica S.A. } \\
(70,8 \%)\end{array}$} & \multicolumn{2}{|c|}{ Fras-le SA $(79,4 \%)$} \\
\hline & \multicolumn{2}{|c|}{ Aços Vilares S.A. $(67,5 \%)$} & \multicolumn{2}{|c|}{$\begin{array}{l}\text { CESP - Cia. Energética de SP } \\
\qquad(69,0 \%)\end{array}$} & \multicolumn{2}{|c|}{$\begin{array}{c}\text { Suzano Papel e Celulose S.A. } \\
(74,8 \%)\end{array}$} \\
\hline & \multicolumn{2}{|c|}{$\begin{array}{c}\text { CESP - Cia. Energética de SP } \\
(64,1 \%)\end{array}$} & \multicolumn{2}{|c|}{ Braskem $(55,9 \%)$} & \multicolumn{2}{|c|}{$\begin{array}{c}\text { Cia. Distribuidora de Gás do } \\
\operatorname{RJ}(73,2 \%)\end{array}$} \\
\hline
\end{tabular}

Nota. ${ }^{\mathrm{a}} \mathrm{QICC}=84,9$ contra 485,2 do modelo base; ${ }^{\mathrm{b}} \mathrm{QICC}=117,7$ contra 707,9 do modelo base; ${ }^{\mathrm{C}} \mathrm{QICC}=79,3$ contra 259,3 do modelo base.

$* \mathrm{p} \leq 0,05 ; * \mathrm{p} \leq 0,01$

Seguindo o modelo teórico proposto na Figura 2, que sugere efeitos anuais das variáveis independentes sobre as mudanças estratégicas para cada empresa em seus respectivos setores, constatase que os dados foram melhor ajustados para mudança de clientes $\left(\mathrm{R}^{2}\right.$ médio $\left.=23,0 \%\right)$, mudança no conselho de administração $\left(\mathrm{R}^{2}\right.$ médio $\left.=21,7 \%\right)$ e mudança de produtos $\left(\mathrm{R}^{2}\right.$ médio $\left.=16,7 \%\right)$, respectivamente. A Tabela 3 apresenta os resultados, incluindo exemplos de empresas que obtiveram maior impacto neles. Percebe-se que os efeitos defasados da lucratividade foram impactantes apenas 
sobre a mudança do conselho de administração, sendo t-1 com efeito positivo e t-2 negativo. Dessa maneira, no agregado, quanto maior a lucratividade, maior a mudança do conselho a longo prazo, com os efeitos positivos maiores do que os negativos. Também, observa-se que o efeito defasado em t- 1 do crescimento das vendas teve impacto positivo sobre a mudança de cliente e efeito negativo sobre a mudança do conselho de administração.

Outrossim, é possível notar que o aumento do valor de mercado da empresa em t-2 tem impacto positivo sobre a mudança de produtos e sobre a mudança do conselho de administração, com maior força sobre o primeiro. Finalmente, quanto mais desconcentrado o setor no período t-2, mais ele propicia a mudança de clientes. Simultaneamente, quanto mais desconcentrado o setor em t-2, menos ele propicia a mudança do conselho de administração, mas há efeito contrário em t-1, de forma que o último anula o efeito do anterior.

Tabela 3

Impacto do Desempenho Financeiro sobre a Mudança Estratégica

\begin{tabular}{|c|c|c|c|c|c|c|}
\hline \multirow{2}{*}{$\begin{array}{l}\text { Variável } \\
\text { preditora }\end{array}$} & \multicolumn{2}{|c|}{ Mudança estrat. de PRO. ${ }^{a}$} & \multicolumn{2}{|c|}{ Mudança estrat. de CLI ${ }^{b}$} & \multicolumn{2}{|c|}{ Mudança estrat. do CAD $^{c}$} \\
\hline & Estimativa & Erro padrão & Estimativa & Erro padrão & Estimativa & Erro padrão \\
\hline $\mathrm{ROA}(\mathrm{t}-1)$ & 0,50 & 0,41 & $-0,27$ & 0,24 & $0,77 * *$ & 0,15 \\
\hline $\mathrm{ROA}(\mathrm{t}-2)$ & $-0,37$ & 0,25 & 0,06 & 0,10 & $-0,12 *$ & 0,06 \\
\hline $\operatorname{VEN}(\mathrm{t}-1)$ & 0,02 & 0,15 & $0,18^{*}$ & 0,09 & $-0,13^{*}$ & 0,06 \\
\hline $\operatorname{VEN}(\mathrm{t}-2)$ & $-0,05$ & 0,07 & $-0,06$ & 0,07 & $-0,01$ & 0,05 \\
\hline $\operatorname{VAM}(\mathrm{t}-1)$ & $-0,48$ & 0,29 & $-0,22$ & 0,25 & $-0,41$ & 0,21 \\
\hline $\operatorname{VAM}(\mathrm{t}-2)$ & $1,27 * *$ & 0,38 & 0,23 & 0,21 & $0,23 * *$ & 0,08 \\
\hline DESCON & $-0,40$ & 0,78 & 0,20 & 0,64 & $-0,77$ & 0,48 \\
\hline $\operatorname{DESCON}(\mathrm{t}-1)$ & 0,05 & 0,56 & $-0,10$ & 0,51 & $2,65^{* *}$ & 0,41 \\
\hline $\operatorname{DESCON}(\mathrm{t}-2)$ & 0,12 & 0,45 & $0,93 *$ & 0,46 & $-2,63 * *$ & 0,22 \\
\hline $\mathrm{R}^{2}$ Linear médio & \multicolumn{2}{|c|}{$16,70 \%$} & \multicolumn{2}{|c|}{$23,00 \%$} & \multicolumn{2}{|c|}{$21,70 \%$} \\
\hline \multirow{3}{*}{$\begin{array}{l}\text { Três empresas } \\
\text { com maior } \\
\text { impacto }\left(\mathrm{R}^{2}\right. \\
\text { linear) em seus } \\
\text { respectivos } \\
\text { setores }\end{array}$} & \multicolumn{2}{|c|}{$\begin{array}{c}\text { CESP - Cia. Energética de SP } \\
\qquad(87,4 \%)\end{array}$} & \multicolumn{2}{|c|}{$\begin{array}{c}\text { CEB - Cia Energética de } \\
\text { Brasília S.A. }(92,8 \%)\end{array}$} & \multicolumn{2}{|c|}{$\begin{array}{l}\text { Suzano Papel e Celulose S.A. } \\
(75,0 \%)\end{array}$} \\
\hline & \multicolumn{2}{|c|}{$\begin{array}{c}\text { Suzano Papel e Celulose S.A. } \\
(66,9 \%)\end{array}$} & \multicolumn{2}{|c|}{$\begin{array}{c}\text { Suzano Papel e Celulose S.A. } \\
(86,7 \%)\end{array}$} & \multicolumn{2}{|c|}{$\begin{array}{l}\text { Fosfértil - Fertilizantes } \\
\text { Fosfatados S.A. }(56,0 \%)\end{array}$} \\
\hline & \multicolumn{2}{|c|}{ Elekeiroz S/A $(47,8 \%)$} & \multicolumn{2}{|c|}{$\begin{array}{l}\text { Fosfértil - Fertilizantes } \\
\text { Fosfatados S.A. }(77,3 \%)\end{array}$} & \multicolumn{2}{|c|}{$\begin{array}{l}\text { Petrobrás - Petróleo } \\
\text { Brasileiro S.A. }(37,0 \%)\end{array}$} \\
\hline
\end{tabular}

Nota. ${ }^{\mathrm{a}} \mathrm{QICC}=148,2$ contra 9.019,2 do modelo base; ${ }^{\mathrm{b}} \mathrm{QICC}=126,3$ contra $6.677,0$ do modelo base; ${ }^{\mathrm{C}} \mathrm{QICC}=89,3$ contra 250,7 do modelo base.

$* \mathrm{p} \leq 0,05 ; * * \mathrm{p} \leq 0,01$

\section{Discussão}

Como um todo, os resultados revelam que os problemas empresariais de Miles e Snow (2003), representados por mudança de produtos e de clientes no curto e no longo prazos, afetam fortemente a lucratividade e levemente o valor de mercado. Igualmente, os problemas administrativos dos mesmos autores, representados por mudança do conselho de administração, afetam todos os três indicadores de desempenho financeiro de empresas brasileiras de capital aberto, independentes da concentração do 
setor. Essa concentração, independentemente das mudanças estratégicas, afeta estruturalmente, de forma corrente e defasada, todos os indicadores, sendo incentivadora do crescimento e do valor de mercado e inibidora da lucratividade. Portanto ela oferece o contexto para geração ou aniquilamento das eficiências da competição empresarial (Bruce \& Marginson, 2013).

Ademais, os desempenhos financeiros anteriores, independentes da concentração do setor, alteram os problemas empresariais e administrativos de Miles e Snow (2003), de forma mais nítida sobre o último do que em relação ao primeiro. Os efeitos defasados da concentração do setor, independente dos desempenhos financeiros anteriores, também, afetam as mudanças estratégicas de clientes e do conselho de administração, corroborando seu incentivo sobre a lealdade de cliente que gera mais receita (Wright \& Riebe, 2010) e criando condições para mudança do conselho devido à baixa importância dos apontamentos do CEO, quando este faz parte daquele (Knyazeva et al., 2009).

Especificamente, com referência aos impactos sobre o desempenho financeiro, uma mudança estratégica dos produtos aumenta a lucratividade, possivelmente, porque é decorrente de uma adaptação bem-sucedida no mercado (Calantone et al., 2004) - mesmo que numa perspectiva dinâmica, essa mudança não atenda mais o mercado em tempo corrente (Calantone et al., 2006). Já a mudança de clientes de maior receita, geralmente, tem efeito negativo de longo prazo sobre a lucratividade e o valor da empresa, confirmando os princípios de retenção de clientes favoritos como uma dimensão do valor deles para a sustentabilidade empresarial (Ramaswami et al., 2009).

As mudanças no conselho de administração em empresas brasileiras de capital aberto geram efeitos correntes e defasados positivos no crescimento da empresa provavelmente para se adaptar ao ambiente (Pugliese et al., 2009) e beneficiar empregados e executivos (David et al., 2010). Como houve efeito negativo de longo prazo sobre o valor de mercado, isso pode ser uma evidência de que essa mudança ocorra em razão da falta de engajamento dos conselheiros anteriores (Minichilli et al., 2009) e que novos membros aconselham aumentar a lucratividade a curto prazo (Fahlenbrach, Low, \& Stulz, 2010), mas, por serem inexperientes, podem não gerar resultados positivos de longo prazo (Minichilli et al., 2009).

Especialmente com relação aos impactos sobre as mudanças estratégicas, os resultados oferecem luz a uma área subpesquisada pela academia de estratégia empresarial, a saber: efeito do desempenho financeiro sobre as atividades empresariais. Assim, bons resultados financeiros permitem reforçar o processo contínuo de mudança para se adaptar ao ambiente também em mutação (Vella \& Foxall, 2011). Este trabalho demonstra que comumente bons desempenhos na lucratividade e no valor de mercado em anos passados incentivam a mudança do conselho, ofertando suporte de que ou (a) os conselheiros deixam de cumprir seu papel básico, possivelmente devido ao baixo monitoramento (Pugliese et al., 2013); ou (b) há baixa recompensa para o CEO, se este fizer parte do conselho; ou (c) há melhores aquisições de empresas e melhores desinvestimentos, que, muitas vezes, alteram membros do conselho (Fischer et al., 2009). Esta pesquisa não oferece luz a qual dessas alternativas ocorre, mas todas elas são explicações plausíveis dos achados.

A presente pesquisa também assinala que o crescimento prévio de vendas da empresa é um incentivador de alterações de clientes mais rentáveis, fator este que diminui sua dependência em relação a alguns clientes prévios não rentáveis, corroborando os achados de Bijmolt et al. (2010). Além disso, com base na proposta teórica de Cyert e March (1963), o crescimento em vendas leva à estabilização do conselho de administração, possivelmente do tipo relacional (David et al., 2010), que tende a reforçar ações dos executivos e funcionários e, assim, perpetua-se por não criar conflitos.

Por fim, esta pesquisa oferece evidência de que o alto valor de mercado incentiva a substituição de produtos que geram mais receita da empresa, sugerindo haver capitalização para a troca de recursos necessários com o fim de inovações e adaptações dos produtos no mercado (Sood \& Tellis, 2009). 


\section{Considerações Finais}

O objetivo deste artigo foi analisar a dinâmica entre o desempenho financeiro e as mudanças estratégicas em empresas brasileiras de capital aberto, controlando a concentração industrial. De modo geral, os resultados apontam que há efeitos reforçadores de um sobre o outro, permitindo fazer inferências causais - boas mudanças estratégicas conduzem ao bom desempenho financeiro competitivo (melhor do que de outras empresas do mesmo setor) - e este produz novas mudanças que podem estimular ou desestimular futuros desempenhos financeiros. Ao longo desse processo, uma empresa pode aumentar (ou reduzir) sua lucratividade, crescimento ou valor no mercado e, consecutivamente, alterar suas ofertas de mercado (receitas vindas do portfólio de produtos), sua base de clientes (dependência de receita de clientes) e seus conselheiros de administração (estrutura e volume do conselho).

Especificamente, a pesquisa demonstra que: (a) todas as mudanças estratégicas investigadas no trabalho, em especial de longo prazo, alteram a lucratividade, sendo a mudança de produto positiva e as restantes negativas; (b) as mudanças do conselho de administração geram efeitos positivos de curto e de longo prazos sobre o crescimento em vendas e efeitos negativos sobre o valor de mercado; (c) a mudança percentual de receitas vindas de cada cliente é maléfica para a lucratividade e para o valor da empresa; (d) a concentração setorial a longo prazo é um contexto desestimulador para a lucratividade e incentivador para o crescimento em vendas e valor de mercado; (e) o aumento do valor de mercado da empresa estimula a longo prazo as mudanças de produto e do conselho de administração; (f) o crescimento em vendas a longo prazo estimula a mudança de cliente e a permanência dos conselheiros de administração; (g) o aumento da lucratividade a longo prazo, em termos gerais, estimula a troca do conselho de administração; e (h) a desconcentração setorial a longo prazo desestimula a permanência de clientes na empresa e seu efeito no conselho de administração é antagônico, ora estimulando a troca, ora desestimulando-a.

Esta pesquisa abre caminhos para que futuras investigações sobre desempenho financeiro empresarial sejam realizadas com uma perspectiva evolucionista, ao considerar atividades empresariais $\rightarrow$ desempenho financeiro e desempenho financeiro $\rightarrow$ atividades empresariais. Apesar da riqueza de informações, a não obrigatoriedade de preenchimento adequado do formulário de produtos e mercados das informações anuais da CVM impossibilitou que se conseguisse um tamanho de amostra maior de sociedades anônimas, sendo uma limitação deste trabalho.

Nem todas as empresas da amostra apresentaram os efeitos aqui demonstrados, constituindo outra limitação no que se refere à generalização dos resultados. Ademais, não foram testadas situações em que há interações entre as variáveis. De acordo com o referencial teórico, algumas relações de interação aparecem como possíveis explicações para os casos em que se encontra uma relação fraca entre variáveis estratégicas e desempenho.

Uma das vantagens do método aqui utilizado por meio de painel temporal de empresas por setor é que não há comparação de empresas em setores diferentes, o que inviabilizaria inferências de causa e consequência. Todas as análises foram desenvolvidas dentro das empresas, ao longo do tempo (withinenterprise), período em que se implementaram as mudanças estratégicas, e o seu próprio desempenho financeiro competitivo foi checado em momentos posteriores. Aconselha-se que futuras pesquisas na área de estratégia empresarial sejam conduzidas dessa maneira para que possam haver inferências de causalidade.

\section{Nota}

\footnotetext{
${ }^{1}$ As opiniões expressas neste trabalho são exclusivamente dos autores e não refletem, necessariamente, a visão de instituições às quais estejam vinculados.
} 


\section{Referências}

Andrade, L. P., Salazar, G. T., Calegário, C. L., \& Silva, S. S. (2009). Governança corporativa: uma análise da relação do conselho de administração com o valor de mercado e o desempenho das empresas brasileiras. Revista de Administração Mackenzie, 10(4), 4-31. doi: 10.1590/S167869712009000400002

Araújo, A. M., \& Assaf, A., Neto (2003). A contabilidade tradicional e a contabilidade baseada em valor. Revista Contabilidade \& Finanças, 14(33), 16-32. doi: 10.1590/S151970772003000300002

Ballinger, G. A. (2004). Using generalized estimating equations for longitudinal data analysis. Organizational Research Methods, 7(2), 127-150. doi: 10.1177/1094428104263672

Bharadwaj, S. G., \& Varadarajan, R. (2005). Toward an integrated model of business performance. In N. Malhotra (Ed.), Review of marketing research, 1, (pp. 207-244). New York: M. E. Sharpe. doi: 10.1108/S1548-6435(2004)0000001009

Bijmolt, T. H., Leeflang, P. S., Block, F., Eisenbeiss, M., Hardie, B. G., Lemmens, A., \& Saffert, P. (2010). Analytics for customer engagement. Journal of Service Research, 13(3), 341-356. doi: $10.1177 / 1094670510375603$

Brito, E. P., Brito, L. A., \& Morganti, F. (2009). Inovação e o desempenho empresarial: lucro ou crescimento? RAE Eletrônica, 8(1). Recuperado de $\mathrm{http} / / / \mathrm{www}$. rae.com.br/eletronica/index.cfm?FuseAction=Artigo\&ID=5232\&Secao=ARTIGOS $\&$ Volume $=8 \&$ Numero=1\&Ano=2009. doi: 10.1590/S1676-56482009000100007

Bruce, A., \& Marginson, D. (2013). Power, not fear: a collusion-based account of betting market inefficiency. International Journal of the Economics of Business, 21(1), 77-97. doi: $10.1080 / 13571516.2013 .782982$

Calantone, R. J., Cavusgil, S. T., Schmidt, J. B., \& Shin, G. C. (2004). Internationalization and the dynamics of product adaptation: an empirical investigation. Journal of Product Innovation Management, 21(3), 185-198. doi: 10.1111/j.0737-6782.2004.00069.x

Calantone, R. J., Harmancioglu, N., \& Droge, C. (2010). Inconclusive innovation “returns": a metaanalysis of research on innovation in new product development. Journal of Product Innovation Management, 27(7), 1065-1081. doi: 10.1111/j.1540-5885.2010.00771.x

Calantone, R. J., Kim, D., Schmidt, J. B., \& Cavusgil, S. T. (2006). The influence of internal and external firm factors on international product adaptation strategy and export performance: a three-country comparison. Journal of Business Research, 59(2), 176-185. doi: 10.1016/j.jbusres.2005.05.001

Capon, N., Farley, J. U., \& Hoenig, S. (1990). Determinants of financial performance: a meta-analysis. Management Science, 36(10), 1143-1159.

Certo, S. T., Lester, R. H., Dalton, C. M., \& Dalton, D. R. (2006). Top management teams, strategy and financial performance: a meta-analytic examination. Journal of Management Studies, 43(4), 813839. doi: 10.1111/j.1467-6486.2006.00612.x

Chandler, A. D. (1962). Strategy and structure: chapters in the history of the American industrial enterprise. Chicago: Massachusetts Institute of Tecnology.

Combs, J. G., Crook, T. R., \& Shook, C. L. (2005). The dimension of organizational performance and its implications for strategic management research. In D. J. Ketchen \& D. D. Bergh (Eds.), Research methodology in strategy and management (pp. 259-286). San Diego: Elsevier. 
Cyert, R. M., \& March, J. G. (1963). A behavioral theory of the firm. Englewood Cliffs: Prentice-Hall.

David, P., O'Brien, J. P., Yoshikawa, T., \& Delios, A. (2010). Do shareholders or stakeholders appropriate the rents from corporate diversification? The influence of ownership structure. Academy of Management Journal, 53(3), 636-654. doi: 10.5465/AMJ.2010.51469005

Fahlenbrach, R., Low, A., \& Stulz, R. M. (2010). Why do firms appoint CEOs as outside directors? Journal of Financial Economics, 97(1), 12-32. doi: 10.1016/j.jfineco.2010.01.003

Fischer, P. E., Gramlich, J. D., Miller, B. P., \& White, H. D. (2009). Investor perceptions of board performance: evidence from uncontested director elections. Journal of Accounting and Economics, 48(2), 172-189. doi: 10.1016/j.jacceco.2009.09.002

Furrer, O., Thomas, H., \& Goussevskaia, A. (2008). The structure and evolution of the strategic management field: a content analysis of 26 years of strategic management research. International Journal of Management Reviews, 10(1), 1-23. doi: 10.1111/j.1468-2370.2007.00217.x

Gee, R., Coates, G., \& Nicholson, M. (2008). Understanding and profitably managing customer loyalty. Marketing Intelligence \& Planning, 26(4), 359-374. doi: 10.1108/02634500810879278

Giroud, X., \& Mueller, H. M. (2011). Corporate governance, product market competition, and equity prices. The Journal of Finance, 66(2), 563-600. doi: 10.1111/j.1540-6261.2010.01642

Gitman, L. J. (2004). Princípios de administração financeira (10a ed.). São Paulo: Pearson.

Greve, H. R. (2007). Exploration and exploitation in product innovation. Industrial \& Corporate Change, 16(5), 945 - 975. doi: 10.1093/icc/dtm013

Gupta, P. D., Guha, S., \& Krishnaswami, S. S. (2013). Firm growth and its determinants. Journal of Innovation and Entrepreneurship, 2(15), 1-15. doi: 10.1186/2192-5372-2-15

Hallowell, R. (1996). The relationships of customer satisfaction, customer loyalty, and profitability: an empirical study. International Journal of Service Industry Management, 7(4), 27-42. doi: $10.1108 / 09564239610129931$

Haynes, K. T., \& Hillman, A. (2010). The effect of board capital and CEO power on strategic change. Strategic Management Journal, 31(11), 1145-1163. doi: 10.1002/smj.859

Hill, M. D., Kelly, G. W., \& Highfield, M. J. (2010). Net operating working capital behavior: a first look. Financial Management, 39(2), 783-805. doi: 10.1111/j.1755-053X.2010.01092.x

Hitt, M. A., Boyd, B. K., \& Li, D. (2004). The state of strategic management research and vision of the future. In D. J. Ketchen \& D. D. Bergh (Eds.), Research methodology in strategy and management (pp. 1-31). Greenwich: JAI Press.

Hutzschenreuter, T., \& Israel, S. (2009). A review of empirical research on dynamic competitive strategy. International Journal of Management Reviews, 11(4), 421-461. doi: 10.1111/j.14682370.2008.00246.x

Ketchen, D., Jr., \& Palmer, T. B. (1999). Strategic responses to poor organizational performance: a test of competing perspectives. Journal of Management, 25(5), 683-706. doi: 10.1016/S01492063(99)00021-5

Ketchen, D., Jr., Snow, C. C., \& Hoover, V. L. (2004). Research on competitive dynamics: recent accomplishments and future challenges. Journal of Management, 30(6), 779-804. doi:10.1016/j.jm.2004.06.002 
Knyazeva, A., Knyazeva, D., \& Raheja, C. (2009). The benefits of focus vs. heterogeneity: an analysis of corporate boards [Unpublished working paper]. University of Rochester, Rochester, NY, EUA.

Labianca, G., Fairbank, J. F., Andrevski, G., \& Parzen, M. (2009). Striving toward the future: aspirationperformance discrepancies and planned organizational change. Strategic Organization, 7(4), 433466. doi: 10.1177/1476127009349842

Lebas, M. J. (1995). Performance measurement and performance management. International Journal of Product in Economics, 41(1-3), 23-35. doi: 10.1016/0925-5273(95)00081-X

Levitt, S. D., \& List, J. A. (2009). Field experiments in economics: the past, the present, and the future. European Economic Review, 53(1), 1-18. doi: 10.1016/j.euroecorev.2008.12.001

Miles, R. E., \& Snow, C. C. (2003). Organizational strategy, structure, and process. Stanford: Stanford University Press.

Minichilli, A., Zattoni, A., \& Zona, F. (2009). Making boards effective: an empirical examination of board task performance. British Journal of Management, 20(1), 55-74. doi: 10.1111/j.14678551.2008.00591.x

Nelson, R. R., \& Winter, S. G. (2005). Uma teoria evolucionária da mudança econômica. Campinas: Unicamp.

Organização para a Cooperação Econômica e Desenvolvimento. (2012). Manual de Oslo: diretrizes para coleta e interpretação de dados sobre inovação. Recuperado de http://download.finep.gov.br/dcom/brasil_inovador/arquivos/manual_de_oslo/prefacio.html

Petersen, J. A., McAlister, L., Reibstein, D. J., Winer, R. S., Kumar, V., \& Atkinson, G. (2009). Choosing the right metrics to maximize profitability and shareholder value. Journal of Retailing, 85(1), 95-111. doi: 10.1016/j.jretai.2008.11.004

Pettigrew, A. M. (2012). Context and action in the transformation of the firm: a reprise. Journal of Management Studies, 49(7), 1304-1328. doi: 10.1111/j.1467-6486.2012.01054.x

Pugliese, A., Bezemer, P. J., Zattoni, A., Huse, M., Van den Bosch, F. A., \& Volberda, H. W. (2009). Boards of directors' contribution to strategy: a literature review and research agenda. Corporate Governance: an International Review, 17(3), 292-306. doi: 10.1111/j.1467-8683.2009.00740.x

Pugliese, A., Minichilli, A., \& Zattoni, A. (2013). Integrating agency and resource dependence theory: firm profitability, industry regulation, and board task performance. Journal of Business Research, 28(3), 383-396. doi: 10.1016/j.jbusres.2013.05.003

Quigley, T. J., \& Hambrick, D. C. (2012). When the former CEO stays on as board chair: effects on successor discretion, strategic change, and performance. Strategic Management Journal, 33(7), 834-859. doi: 10.1002/smj.1945

Quinn, J. B. (1978). Strategic change: logical incrementalism. Sloan Management Review, 20(1), 7-21.

Ramaswami, S. N., Srivastava, R. K., \& Bhargava, M. (2009). Market-based capabilities and financial performance of firms: insights into marketing's contribution to firm value. Journal of the Academy of Marketing Science, 37(2), 97-116. doi: 10.1007/s11747-008-0120-2

Ryals, L. J., \& Knox, S. (2005). Measuring risk-adjusted customer lifetime value and its impact on relationship marketing strategies and shareholder value. European Journal of Marketing, 39(5), 456-472. doi: 10.1108/03090560510590665

Simonsen, M. H. (1979). Teoria microeconomica: teoria da concorrência perfeita, teoria da concorrência imperfeita (Vol. II, 2a ed.). Rio de Janeiro: FGV. 
Sood, A., \& Tellis, G. J. (2009). Do innovations really pay off? Total stock market returns to innovation. Marketing Science, 28(3), 442-456. doi: 10.1287/mksc.1080.0407

Sorenson, O. (2000). Letting the market work for you: an evolutionary perspective on product strategy. Strategic Management Journal, 21(5), 577-592. doi: 10.1002/(SICI)10970266(200005)21:5<577::AID-SMJ105>3.0.CO;2-C

Sundaram, A. K., \& Inkpen, A. C. (2004). The corporate objective revisited. Organization Science, 15(3), 350-363. doi: 10.1287/orsc. 1040.0068

Vella, K. J., \& Foxall, G. R. (2011). The marketing firm: economic psychology of corporate behaviour. Northampton, Massachusetts: Edward Elgar Publishing.

Venkatraman, N., \& Ramanujam, V. (1986). Measurement of business performance in strategy research: a comparison of approaches. Academy of Management Review, 11(4), 801-814. doi: 10.5465/AMR.1986.4283976

Vrontis, D., Thrassou, A., \& Lamprianou, I. (2009). International marketing adaptation versus standardisation of multinational companies. International Marketing Review, 26(4/5), 477-500. doi: 10.1108/02651330910971995

Westphal, J. D., \& Fredrickson, J. W. (2001). Who directs strategic change? Director experience, the selection of new CEOs, and change in corporate strategy. Strategic Management Journal, 22(12), 1113-1137. doi: 10.1002/smj.205

Wright, M., \& Riebe, E. (2010). Double jeopardy in brand defection. European Journal of Marketing, 44(6), 860-873. doi: 10.1108/03090561011032748 